\title{
Designing fuzzy expert system for creating and ranking of tourism scenarios using fuzzy AHP method
}

\author{
Zohreh Nikkhah $^{\mathrm{a}}$ and Ahmad Makui ${ }^{\mathrm{b}}$
}

${ }^{a}$ Department of Industrial Engineering, Science \& Research Branch, Islamic Azad University, Tehran, Iran ${ }^{b}$ Department of Industrial Engineering, Iran University of Science \& Technology, Tehran, Iran

\begin{tabular}{|c|c|}
\hline A R T I C L E I N F O & A B S T R A C T \\
\hline $\begin{array}{l}\text { Article history: } \\
\text { Received } 25 \text { June } 2010 \\
\text { Received in revised form } \\
14 \text { October } 2010 \\
\text { Accepted } 14 \text { October } 2010 \\
\text { Available online } \\
14 \text { October } 2010 \\
\text { Keywords: } \\
\text { Analytical hierarchy process } \\
\text { Mamdani fuzzy method }\end{array}$ & $\begin{array}{l}\text { One of the most important activities of tour and travel agencies is to select the appropriate tour } \\
\text { configuration. There are normally two primary objectives of season and time period to set a } \\
\text { group of cities called designing tour scenarios. The success of tour scenarios is deeply related to } \\
\text { the experiments and wisdom of the experts and planners in travel agencies. This paper presents } \\
\text { a fuzzy rule decision making to find the suitable set of cities where different possible criteria } \\
\text { are ranked using analytical hierarchy procedure. The proposed model of this paper is applied } \\
\text { for a real-world case study of Iranian tour agency and the results are analyzed under different } \\
\text { circumstances. }\end{array}$ \\
\hline
\end{tabular}

Tourism Industry

Multiple criteria decision making

\section{Introduction}

Travelling and tourism industry is considered the greatest and the most diverse industry in the world. Most countries consider this dynamic industry as the main source of revenue, employment and growth of their private sector and infrastructural development. Similar to other businesses, tourism industry also includes numerous sub-sectors. One of these sub-sectors considered as the fastest growing areas of tourism, must be declared as activities related to tour operating or tour planning agency and the most important part is designing of tourism packages with the following two objectives.

1. Designing tour scenarios

2. Combining the tour scenarios by various factors such as residency, means of transportation and extra services.

\footnotetext{
* Corresponding author. Tel: +982177240541 Ext: 5004

E-mail addresses: amakui@iust.ac.ir (A. Makui), 
The primary focus of this research is on designing tour scenarios. Tour scenario involves the selection of city and the priority of visiting the cities based on two inputs of the season of the year and the duration of stay in the city. There are many cases where an expert determines the details of scenario planning. This could lead to a feasible solution which may or may not be efficient practically. The suitable configuration of cities could be also established using multiple criteria decision making (MCDM) methods such as analytical hierarchy process (AHP). The implementation of AHP could help us perform a pairwise comparison for all different choices and alternatives. The process is normally repeated among various experts either in a team work or through a personal interview. We may also use the concept of fuzzy programming whenever a precise decision cannot be extracted directly. In this paper we propose a method which uses both AHP and fuzzy rules to determine the best combination of a tour configuration. We plan to find all possible scenarios dynamically and the best combinations of scenarios are chosen using AHP method.

One of the issues of a successful tourism is to measure the loyalty of customers to a particular agency. Pearce and Kang (2009) performed a survey on some South Korean people who were visiting Australia and determined some important factors affecting the customers' decision. Allen et al. (2009) performed a survey on Australian people who were visiting different cities and determined two important factors of income and accommodations prices for the frequency of their travelling pattern. One of the important factors in tourism industry is the tour guide which is distributed among travelers (Low et al, 1996). Zhang and Chow (2004) did a survey on tour guides based on 20 attributes and found that 11 attributes helped travelers from China who were visiting Hong Kong pretty well. Nationality is the other factor motivating factor for the people who travel to a particular country. Kozak (2002) performed a pull and push study on some travelers who visited Mallorca and Turkey in the summer of 1998. He analyzed his findings using some statistical tests such as Factor analysis and found that some tourist motives differed between nationalities and place visited. The characterization of hotel destination is another important factor affecting travelers' decision behavior. Ngai and Wat (2003) developed a fuzzy decision making method for choosing suitable hotel and executed their model on various hotels located in Hong Kong. Chou et al. (2008) developed a decision making procedure to find a suitable hotel based on the distance from different places. Using a fuzzy decision making method, they developed a decision support system to help find the suitable hotel. Chen et al. (2010) used the concept of fuzzy programming to forecast the number of tourists who will come to a country. Nikole and Francisco (2006) studied the relationship between the price and the distance that affects hotel selection. This paper is organized as follows. We first develop the proposed model of the paper in section 2. Section 3 is devoted to the implementation of the proposed fuzzy model. Finally conclusion remarks are given at the end to summarize the contribution of the paper.

\section{Definitions}

The following tour scenarios exist.

- Tour scenarios with fixed center: In this travel package, tourist selects a fixed center and constantly moves and visits around the center during the stay. In this scenario, tourist is not benefited by the diversity of residency in different places.

- Circular tour scenario: In this travel package, tourist is benefited by residency in different places and every time passes new passages.

- Tour scenario with open passage: In this plan tourist enters the destination in the target country and moves there to visit different attractions in the cities and even neighboring countries. However tourist does not return to a destination in order to get back to his home country, but moves toward his home country from the last city or country visited. This sort of tour scenario is called tour scenario with open passage. 


\section{Assumptions}

The following assumptions hold.

- The tour plan is designed for Iran.

- The interests and motivation of tourists for the trip is completely clear, and the motivation of the tour members is cultural-historical.

- All tour members are foreigners and plan to stay in tour for 2 to 20 days

- The primary concern of all tourists is to visit historical monuments.

- Historical cities are limited to Isfahan, Tehran, Shiraz, Hamadan, Kermanshah, Kerman, Ahvaz, Tabriz, Mashhad and Yazd.

- Selection of cities does not depend on number of people who form the tour.

- The tour package only serves the first time travelers.

- Circular type of tour scenario has been selected for the tourists.

\section{The Proposed Methodology}

In the proposed method of this paper we need to collect the necessary information from some experts. Therefore we prepare some questionnaire so that we could gather the information using a face-to-face interview. The survey must include the response of some technical and important questions from, at least, five travel agencies. Table 1 summarizes the first set of the necessary information which specifies the priority of the visiting cities.

\section{Table1}

The priority of visiting historical cities

\begin{tabular}{lc}
\hline Historical city & Visiting priority \\
\hline Isfahan & 1 \\
Shiraz & 2 \\
Yazd & 3 \\
Kerman & 4 \\
Tehran & 5 \\
Ahvaz & 6 \\
Hamadan & 7 \\
Mashhad & 8 \\
Kermanshah & 9 \\
Tabriz & 10 \\
\hline
\end{tabular}

Next, we need to specify the number of days we need to visit the cities. Table 2 summarizes the information regarding the minimum number of days that tourists must stay in a city. 


\section{Table 2}

Minimum number of days

\begin{tabular}{ll}
\hline City & Minimum number of days \\
\hline Isfahan & 3 days \\
Shiraz & 2 days \\
Yazd & 2 days \\
Tabriz & 2 days \\
Hamadan & 1 day \\
Mashhad & 1 day \\
Kerman & 2 days \\
Tehran & 2 days \\
Ahvaz & 1 day \\
Kermanshah & 1 day \\
\hline
\end{tabular}

If Tehran is in a tour schedule it must be visited first. Also the cities are classified into three groups: The first group includes Tehran, Isfahan, Shiraz, Kerman, Yazd and Ahvaz. The second group covers Tehran, Hamadan, Kermanshah and Tabriz. The third one only covers Tehran and Mashhad. Note that, since Tehran is the entering and exiting spot, it is repeated in all the tour packages. Since visiting the sites between the cities are very important for visitors, any land travel is normally recommended by customers.

\subsection{Extracting fuzzy rules}

As we explained earlier, there are two important factors in prioritizing the cities. The first one is the amount of time we spend to stay in a city and the second one is the visiting season. Mamdani (1975) is believed the first who used fuzzy logic to approximate reasoning using linguistic synthesis. We use the same procedure as suggested by Mamdani to prioritize different rules. The analysis uses if and then clause. On the if part, two inputs are used and on the then part ten outputs are considered and all possible relationships are joint using the logical term of AND joint and 36 fuzzy if-then combinations are designed. The next step is to classify each group in visiting sequence based on the priority rule of visiting. In order to do this we need to indentify the necessary criteria in selecting the most suitable scenario from the experts' point of view. Finally we need to prioritize the cities based on an AHP method which has three different levels. In the first level, the primary objective is to find the most appropriate tour scenario. The second level considers three criteria of visiting area quality, nationality of tourist and profitability where the profitability criterion is divided into two sub-criteria of extra service sale and direct sale of tour package. The third level considers the following three criteria,

1. Quality of transportation and residential facility

2. Level of access to welfare facility

3. Extra service quality

To perform pairwise comparison we use numbers from one to nine. Whenever we are unable to get a number from expert we use fuzzy numbers given in Table 3. 
Table 3

Fuzzy words and corresponding numbers

\begin{tabular}{llc}
\hline Explanatory notes & Logical term & Numeral value \\
\hline Extremely superior or more significant or more desirable & Extreme & 9 \\
Preference or significance or very strong desirability & Very strong & 7 \\
Preference or significance or strong desirability & Strong & 5 \\
Moderately preferable or more significant or more desirable & Moderate & 3 \\
$\begin{array}{l}\text { Equal preference or significance or desirability } \\
\text { Preferences between the above distances }\end{array}$ & & $2,4,6,8$ \\
\hline
\end{tabular}

\subsection{Inference fuzzy system construction}

The Mamdani fuzzy controller system receives the input terms in terms of logical expression and send the information into the fuzzy inference engine where inputs are defuzzified based on fuzzy rule base. Fig. 1 demonstrates the details of the fuzzy logic interface.

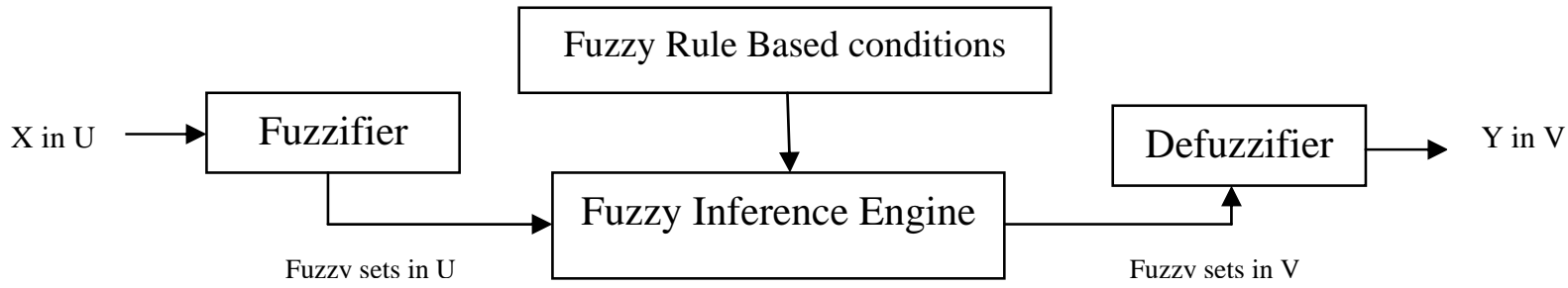

Fig.1. A fuzzy system construction.

In the presented fuzzy system, Mamdani inference method has been used where the AND operation is based on minimization and defuzzification and it is performed by Centeroid Method. The following summarizes the necessary steps of our proposed method.

1. Inputs and outputs of the model: In the present research two inputs of Ttd which stands for residency (number of days) and Season which stands for season of year have been used as inputs. Residency variables changes from 2 to 20 days, and season variables includes four seasons of year (spring, summer, autumn, winter). The output of the model also includes the names and the circumstances of 10 historical cities based on three terms of bad, good and perfect for selection.

2. Attributing membership functions to input and output variables: The membership functions are associated with two variables; number of days and season of year are defined as trapezoidal function. The membership functions associated with the output variables of historical cities are defined as generalized bell membership function. The collection of information is known as database in the field of membership functions of fuzzy variables and domain of fuzzy variables. The objective functions are defined based on the nature of the data and the application of fuzzy toolbox.

3. Designing fuzzy rules base: As we already explained, 36 sets of if-then fuzzy rules are extracted and each one of these if-then rules includes two inputs in prior sector and 10 outputs in the following sector.

4. Combining outputs and forming a single fuzzy set: Since the decisions are based on testing all the rules in fuzzy inference system, it is necessary to combine the rules to make decision. Combination is a process in which all the extracted fuzzy sets from each rule are combined together and produce a single fuzzy set. 
5. Defuzzication: output of model from the previous step is a fuzzy set and it is necessary that the resulted fuzzy value is transformed to a determined spot as the best choice of output fuzzy set.

If the stay duration in Iran is equal to13 days, therefore $\mathrm{Ttd}=13$ and this means we are in $\mathrm{T} 14$ and season of year is equal to 0.494 , by referring to membership functions base, it is observed that this numeric value belongs to both fall and summer. In other words, there is not much difference between late summer and early autumn from climatic point of view, therefore the membership function about the number of days and season of year and historical cities condition are shown in Fig. 2.
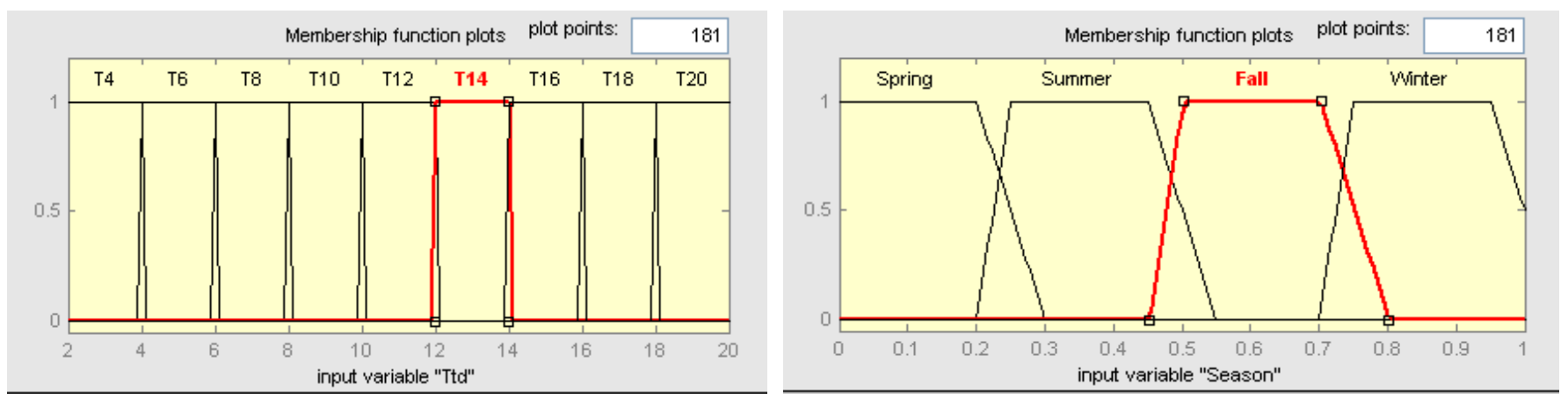

Fig. 2. (a) Number of days membership function Fig. 2. (b) Season of the year membership function

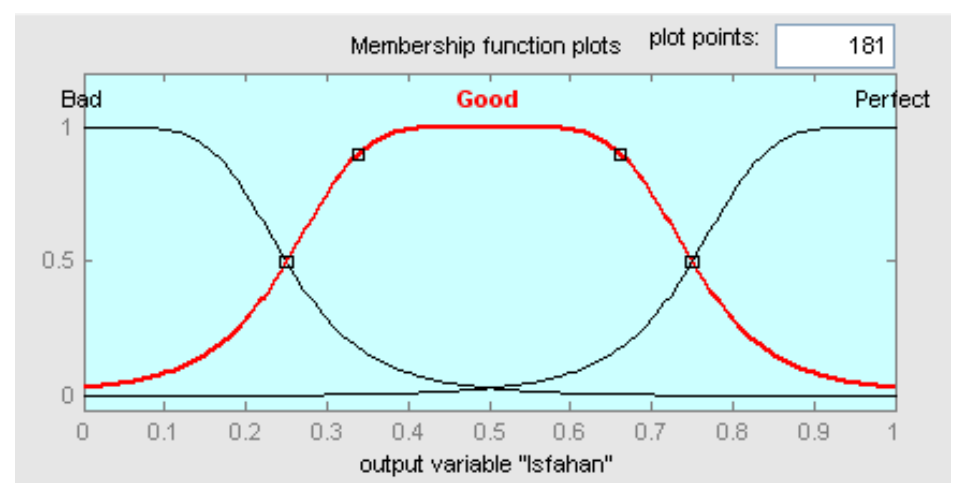

Fig. 2. (c) Condition of cities membership function

The rules in fuzzy system have been extracted using expert people (See Appendix A). Table 4 summarizes the defuzzified values associated with each city.

Table 4

Defuzzified values per historical city

\begin{tabular}{llll}
\hline City & value & City & Value \\
\hline Isfahan & 0.6750 & Kerman & 0.50 \\
Shiraz & 0.6750 & Yazd & 0.6750 \\
Tehran & 0.6750 & Ahvaz & 0.50 \\
Hamadan & 0.6480 & Tabriz & 0.6480 \\
Mashhad & 0.6750 & Kermanshah & 0.6480 \\
\hline
\end{tabular}

Table 5 also shows the details of degree of dependency to three logical words of good, bad and perfect. 
Table 5

Degree of dependency to bad,good and perfect set

\begin{tabular}{lllll}
\hline City & Defuzzified values & $\begin{array}{l}\text { Degree of } \\
\text { dependency to good } \\
\text { set }\end{array}$ & $\begin{array}{l}\text { Degree of } \\
\text { dependency to bad } \\
\text { set }\end{array}$ & $\begin{array}{l}\text { Degree of } \\
\text { dependency to } \\
\text { perfect set }\end{array}$ \\
\hline Isfahan & 0.6750 & 0.85 & 0.0 & 0.15 \\
Shiraz & 0.6750 & 0.85 & 0.0 & 0.15 \\
Tehran & 0.6750 & 0.85 & 0.0 & 0.15 \\
Hamadan & 0.6480 & 1 & 0.0 & 0.9 \\
Mashhad & 0.6750 & 0.85 & 0.0 & 0.15 \\
Kerman & 0.5 & 1 & 0.0 & 0 \\
Yazd & 0.675 & 0.85 & 0.0 & 0.15 \\
Ahvaz & 0.5 & 1 & 0.0 & 0 \\
Tabriz & 0.6480 & 0.1 & 0.0 & 0.9 \\
Kermanshah & 0.6480 & 0.1 & 0.0 & 0.9 \\
\hline
\end{tabular}

Fig. 3 shows the roadmap of fuzzy inference process. Twelve small plots (two yellow plots and ten blue plots) across the top of the figure represent the antecedent and consequent of the first rule. Each rule is a row of plots, and each column is a variable. The first two columns of plots (the eight yellow plots) show the membership functions referenced by the antecedent, or the if-part of each rule. The forty blue plots show the membership functions referenced by the consequent, or the then-part of each rule. The fifth plot in each column of plots represents the aggregate weighted decision for the given inference system. This decision will depend on the input values for the system.

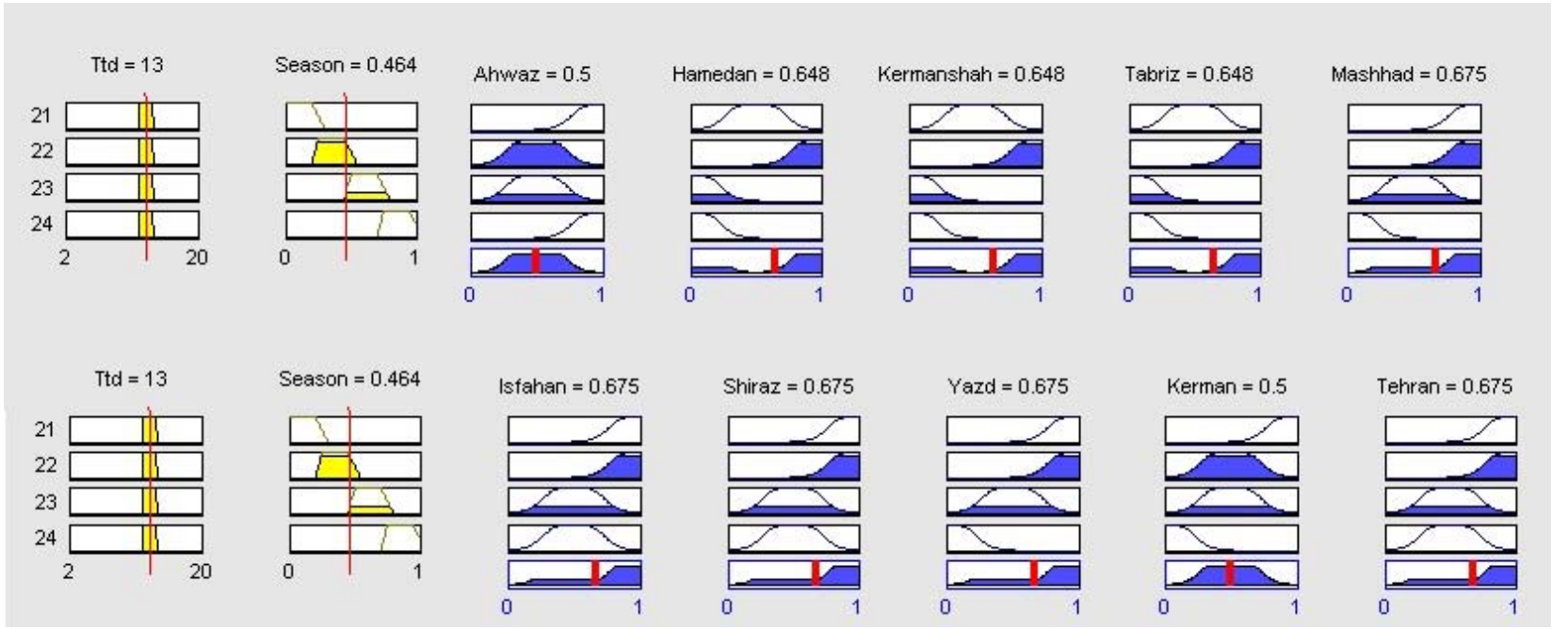

Fig.3. Roadmap of fuzzy inference process

The relationship between the membership function of two inputs and one outputs generate threedimensional plots shown in Fig.4. In this figure the relationships between two inputs and one outputs: residential time and seasons of the year and the output of selected historical cities' circumstance can be observed, simultaneously. It shows the circumstance of Shiraz city considering any number of days and season of year. 


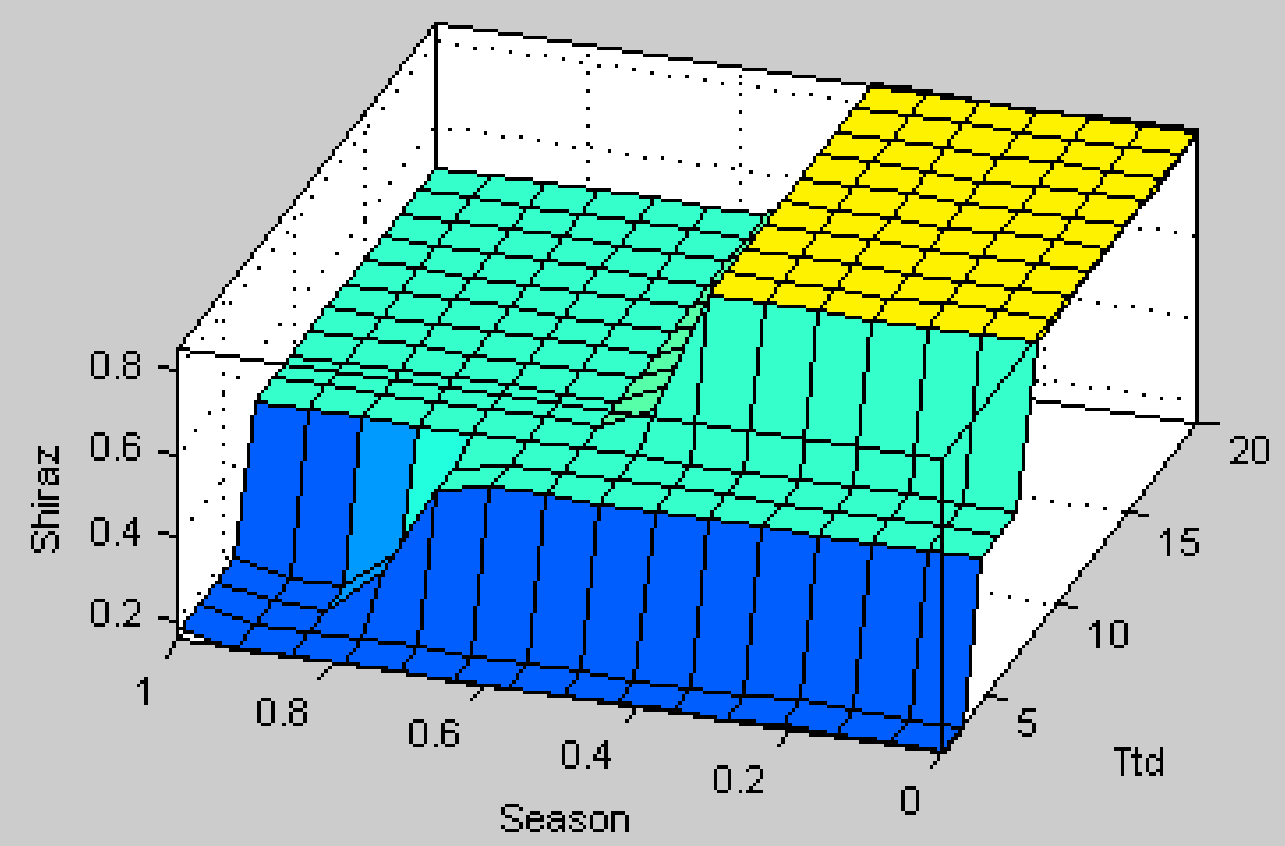

Fig.4. Mapping the inputs to output for the city of Shiraz

As we explained earlier, there are three groups of cities and since Tehran must be included in all three groups we have,

1. Tehran-Shiraz- Isfahan- Kerman- Yazd- Ahvaz

2. Tehran- Hamadan- Tabriz- Kermanshah

3. Tehran- Mashhad

It is also desired that all tourists travel from the first city to the last one by airplane and from the last city they are returned to the nearest one by car and the process continues using busses till all passengers come back to the origin city. The following is the summary of three groups,

1. Tehran- Ahvaz- Shiraz- Kerman- Yazd- Isfahan

2. Tehran- Tabriz- Hamadan- Kermanshah

3. Tehran- Mashhad

Evaluation of extracted scenarios using hierarchical model of AHP(see Fig.5). 


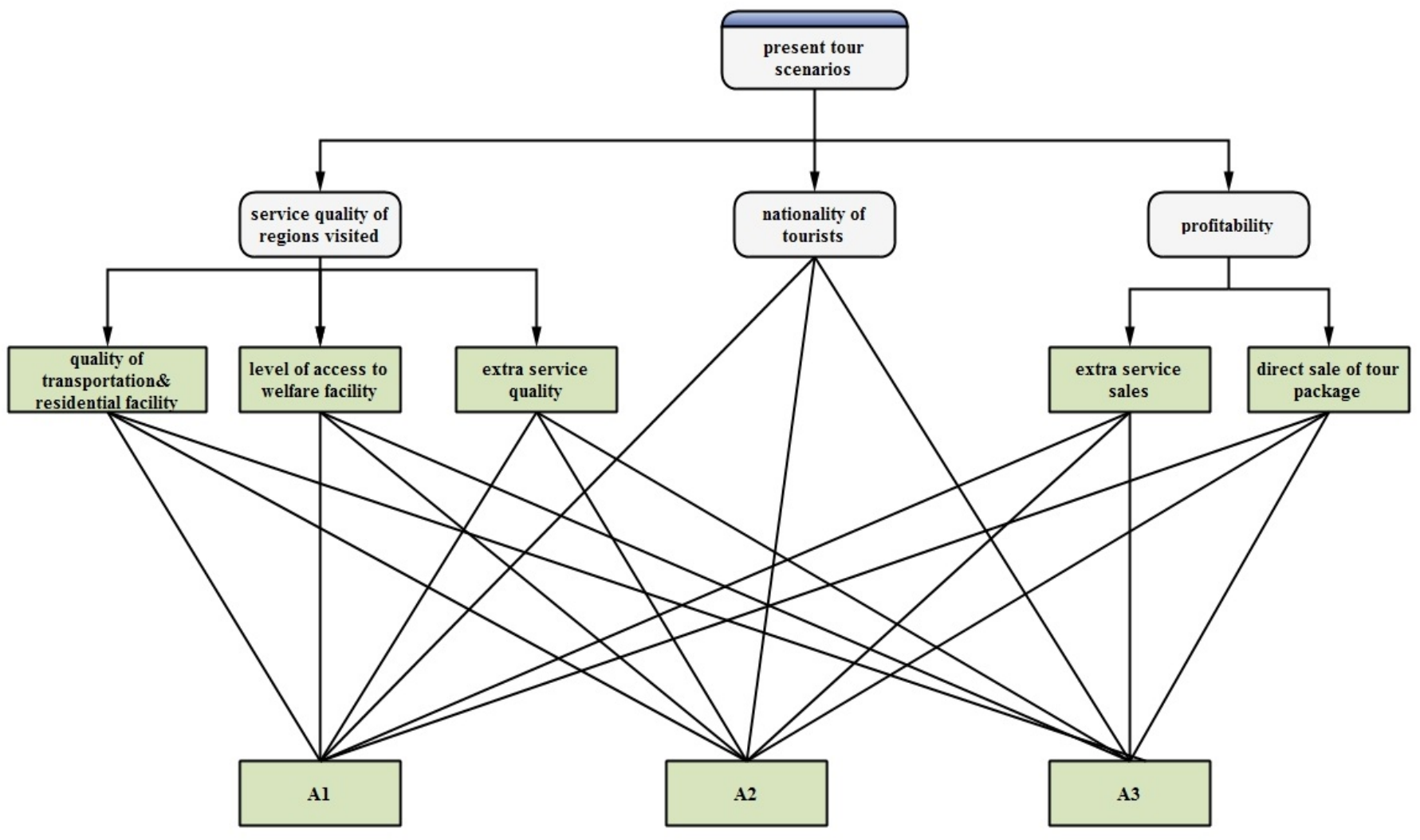

Fig.5.The analytical hierarchical process

The final weights of paired comparison matrices in three presented scenarios:

A1: Tehran- Mashhad

A2: Tehran- Ahvaz- Shiraz- Kerman- Yazd- Isfahan

A3: Tehran- Tabriz- Hamadan- Kermanshah

\section{Table 6}

The final weights extracted from AHP analysis for three attributes

Criterion weight

Quality of residential facilities and transportation

Nationality of tourist 


\section{Table 7}

The final weights related to paired comparison matrices of relationships between sub-criteria

\begin{tabular}{lc}
\hline Sub-criterion & weight \\
\hline Quality of residential facilities and transportation & 0.4551 \\
Access amount to welfare facilities & 0.0544 \\
Quality of extra services & 0.1296 \\
Direct sale of tour package & 0.0222 \\
Extra service sale & 0.0940 \\
\hline
\end{tabular}

The results obtained from the analysis of data can be classified as follows:

1. Results obtained from developing Mamdani fuzzy system indicate that as the red index line in output of model and in input of number of days moves toward right, the number of days tends to increase and the selection of cities with high historical attractions tends to be the best for selection. Also when we consider the season of the year as input we realize that as the red index line moves between the span of spring and summer, selection of significant historical cities from the viewpoint of historical attraction, residential attraction and transportation tends toward good and the best circumstance for selection.

2. The final evaluation of the criteria has been performed by AHP fuzzy model and the results A1 is Tehran- Mashhad, A2 is Tehran- Ahvaz- Shiraz- Kerman- Yazd- Isfahan and A3 is Tehran- Tabriz- Hamadan- Kermanshah. Also the fuzzy utilities preference score are as follows $\mu \mathrm{s}(1)=0.15, \mu \mathrm{s}(2)=1.0$ and $\mu \mathrm{s}(3)=0.9$. Therefore, A2 utility tour scenario is the first preference and A3 tour scenario is the second and A1 tour scenario is the third. Fig.6 shows the results of the ranking fuzzy utility scenarios.

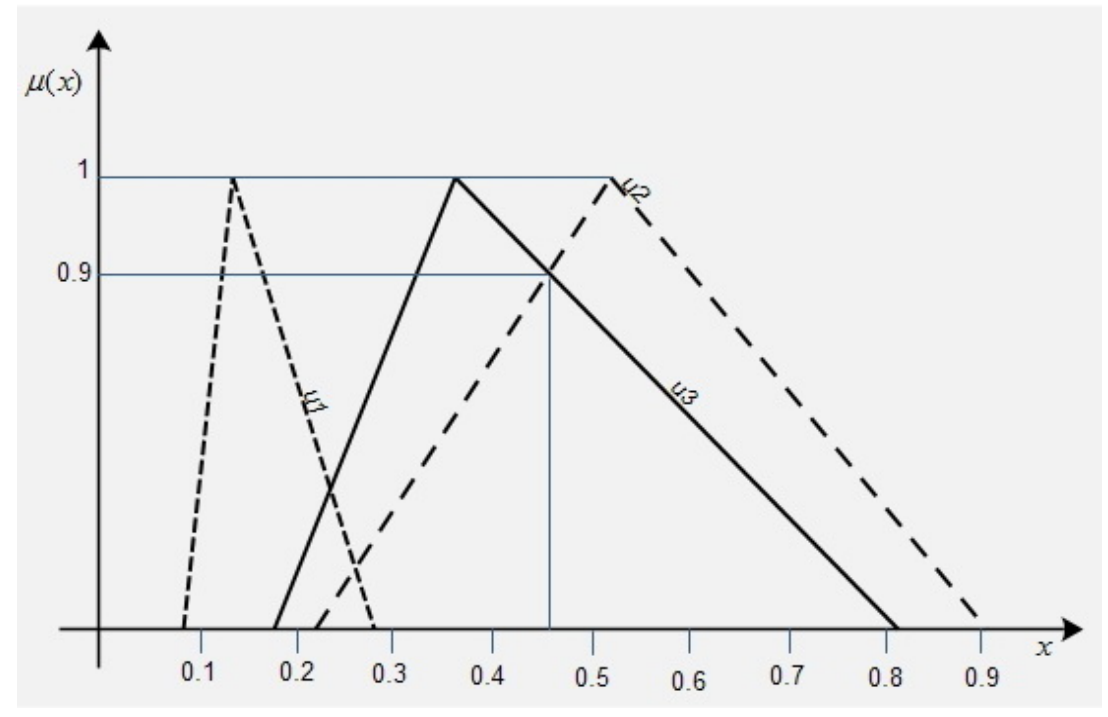

Fig.6. results of ranking fuzzy utility scenarios A1, A2, A3 


\section{Conclusion}

In this paper, we have presented a fuzzy decision making methodology to find the best scenario planning of an Iranian tourist industry. We have considered two primary criteria for designing a methodology which were the number residential and the season of the year. The proposed model of this paper has considered AHP method to prioritize different criteria and using fuzzy numbers we have used Mamdani method to analyze the proposed approach for an empirical study of Iranian case study. The results are also analyzed under various circumstances.

\section{Acknowledgment}

We would like to appreciate the honorary referees' precious opinions and guidance which have had a great influence in improving the article.

\section{Appendix A - fuzzy rules for the time of 13 days and season of year equal to 0.494 .}

1. If(Ttd is T14) and (Season is Spring) then (Isfahan is Perfect)(Shiraz is Perfect)(Yazd is Perfect)(Kerman is Perfect)(Tehran is Perfect)(Ahwaz is Perfect)(Hamedan is Good)(Kermanshah is Good)(Tabriz is Good)(Mashahd is Perfect)

2. If(Ttd is T14) and (Season is Summer) then (Isfahan is Perfect)(Shiraz is Perfect)(Yazd is Perfect)(Kerman is Good)(Tehran is Perfect)(Ahwaz is Good)(Hamedan is Perfect)(Kermanshah is Perefect)(Tabriz is Perefect)(Mashahd is Perfect)

3. If(Ttd is T14) and (Season is full) then (Isfahan is Good)(Shiraz is Good)(Yazd is Good)(Kerman is Good)(Tehran is Good)(Ahwaz is Good)(Hamedan is Bad)(Kermanshah is Bad)(Tabriz is Bad)(Mashahd is Good)

4. If(Ttd is T14) and (Season is Winter) then (Isfahan is Good)(Shiraz is Good)(Yazd is Bad)(Kerman is Bad)(Tehran is Good)(Ahwaz is Perfect)(Hamedan is Bad)(Kermanshah is Bad)(Tabriz is Bad)(Mashahd is Bad)

\section{References}

Pearce, Ph. L., \& Kang, M-h. (2009). The effects of prior and recent experience on continuing interest tourist settings. Annals of Tourism Research, 36(2), 172-190.

Chou, T-Yu., Hsu, Ch-L., \& Chen, M-Ch. (2008). A fuzzy multi-criteria decision model for international tourist hotels location selection. International Journal of Hospitality Management, 27(2), 293-301.

Kozak, M. (2002).Comparative analysis of tourist motivations by nationality and destinations. Tourism Management , 23(3), 221-232.

Allen, D., Yap, Gh., \& Shareef, R. (2009). Modelling interstate tourism demand in Australia: A cointegration approach. Mathematics and Computers in Simulation, 79(9), 2733-2740. 
Zhang, H. Q., \& Ivy, Ch. (2004). Application of importance-performance model in tour guides' performance: evidence from mainland Chinese outbound visitors in Hong Kong, Tourism Management, 25(1), 81-91.

Nikole, J. L., \& Francisco, J. M. (2006). The influence of distance and prices on the choices of tourist destination: The modelling role of motivations. Tourism Management, 27(5) 982-996.

Ngai, E. W. T., \& Wat, F. K. T. (2003). Design and development of a fuzzy expert system for hotel selection, Omega, 31(4), 275-286.

Chen, M-Sh, Ying, L-Ch., \& Pan, M-Ch. (2010). Forecasting tourist arrivals by using the adaptive network-based fuzzy inference system, Expert Systems with Applications, 37(2), 1185-1191.

Low, B. T., Cheng, Ch-H., Wong, K. F., \& Motwani, J. (1996). An expert advisory for the tourism industry, Expert Systems with Applications, $11(1)$, 65-77. Mamdani, E. H. (1975). Application of fuzzy logic to approximate reasoning using linguistic synthesis, IEEE Transactions on Computers, 26(12), 1182-1191. 